\title{
CURRICULUM ELEMENTAL DE METRICA
}

\section{Josep Ferrer * \\ Josep Lluis Ortega}

Escuela Especializada de Nuestra Señora de Meritxell

\section{Currículum elemental de métrica}

Un currículum de métrica tiene como mínimo tres funciones principales en el contexto de una educación especializada. En primer-lugar, tanto para los alumnos que en su vida adulta hayan de desenvolverse autónomamente como para los que hayan de permanecer en instituciones especializadas (residencias y talleres protegidos), parece claro que el conocimiento de los diferentes tipos de medidas que se contemplan en el presente currículum (longitud, temperatura, peso, volumen) ha de resultarles útil en un amplio contexto de actividades cotidianas, que irian desde la compra de diferentes artículos hasta la regulación de termostatos.

En segundo lugar, e igualmente también para unos como para otros, resulta altamente probable que estos conocimientos de medidas sea imprescindible o, al menos, muy útil para la realización de parte de las tareas de trabajo en los que al menos algunos sujetos hayan de intervenir.

Finalmente, y desde una perspectiva puramente académica, el currículum de métrica supone un campo de aplicación funcional de aprendizajes anteriores o simultáneos, como son numeración, operaciones, etc., con todas las ventajas que eso supone para la generalización y mantenimiento de éstos.
Al revisar la literatura de que disponíamos al respecto, consideramos sólo de pasada los currículums (habitualmente sólo fichas intercaladas entre otras referidas a contenidos diversos) de los textos escolares normales. Con éstos resulta difícil alcanzar una estructura realmente exhaustiva, detallada y sistemática.

Afortunadamente, a través de la red ERIC habiamos accedido a tres trabajos diferentes que han resultado suficientemente adecuados, aunque no han dejado de requerir una cierta adaptación que pasamos a comentar a continuación.

El «Elementary Metric Curriculum. Project T.I.M.E. (Timely Implementation of Metric Eduaction)» (ED $171-556$, ED 171-557), es un manual para educadores para la enseñanza del sistema métrico a nivel de escolaridad elemental que incluye objetivos conductuales y actividades para el alumno. Está dividido en dos partes, la primera de las cuales cubre las siguientes temáticas o contenidos: medición lineal, relaciones métrico-decimales, conversiones métricas, geometría, dibujo a escala y capacidad. La segunda trata: capacidad, cálculo de volúmenes y áreas de cilindros y conos, masa, temperatura y conversiones métricas. De este currículum hemos seleccionado sólo algunos tópicos, pero de éstos la mayor parte de objetivos $y$ actividades, aunque hemos hecho el esfuerzo de redefinir los objetivos para

* Dirección del autor: Escuela Especializada de Nuestra Señora de Meritxell. Andorra. 
adaptarlos a la normativa del «Grupo de Investigación en Educación Especial».

"Teaching Measurement to Children: Grades K-6, Revised Edition" (M. L. Borelli, S. Z. Morelli, 1978, ED. 171-947) propone una jerarquía de niveles para la ordenación de los objetivos de métrica referentes a la longitud, distancia, área, volumen, capacidad, masa y temperatura. Una característica peculiar de este modelo es que las primeras experiencias de los alumnos son de naturaleza no numérica, es decir, a base de comparaciones físicas directas. Sólo a continuación se introduce la medición numérica para, finalmente, entrar en el desarrollo del uso de instrumentos. Los objetivos se encuentran secuenciados de la forma-siguiente:

I. Medición no numérica.

A) Comparación directa

1. Adquisición del lenguaje

2. Igualdad-diferencia

3. Relación de orden

4. Selección del valor extremo

s. Seriación.

II. Medición numérica

A) Modelos físicos

I. Aparejamiento y descripción

2. Estimación

3. Relación de orden

4. Seriación

B) Utilización de instrumentos
I. Concepto de calibración
2. Medición
3. Estimación
4. Seriación.

Estos dos textos han sido los más utilizados para la confección del progra- ma actualmente en rodaje. Del primero de ellos hemos sacado prácticamente la totalidad de objetivos (siemprisile unos temas o apartados determinadis, y de actividades. Del segundo hemos añadido algunos objetivos y algunas ideas para la ordenación de éstas dentro del programa.

Un último texto, «Metrics: The Future is Now!" (Community School District I8, Brooklyn, NY, ED. 171-555) trata más bien el problema de la conversión del sistema americano de medida, al sistema decimal. De él hemos extraído algunas actividades que nos han parecido interesantes.

En resumen, hemos podido partir de un material muy organizado y que hemos considerado como bueno. A partir de éste ha sido precisa una remodelación formal del enunciado de los objetivos, reordenar y completar en cierta medida estos objetivos y una adaptación a los niveles presumibles de nuestrós. alumnos. El material de actividades propuestas es bueno, pero requerirá seguramente de un mayor detalle y parsimonia, lo cual supone un proceso de adaptación que ya ha sido iniciado.

El trabajo presente consiste en una exposición ordenada de los objetivos del currículum referentes a cuatro secciones: longitud, temperatura, capacidad y peso. Dentro de cada sección los objetivos están divididos en dos grandes apartados: medición no numérica y medición numérica y enumerados correlativamente. A continuación, se describen las actividades disponibles para cada objetivo de longitud y temperatura, descritas en términos de material, consignas y respuesta esperada del alumno.

\section{CURRICULUM ELEMENTAL DE METRICA-OBJETIVOS}

\section{Longitud}

\section{Medición no numérica}

I. R.: a) dados tres objetos, dos de ellos de igual longitud, el alumno indicará bajo la consigna verbal: "pinta», "señala», «rodea», etc., los iguales. 
b) dados dos objetos claramente diferentes o iguales en longitud, el alumno reconocerá verbalmente si son o no son iguales.

Co.: los objetos presentados serán reales, o representados en dibujos, aparte de la longitud, que variará según cada caso, los objetos tendrán las mismas características.

Cr.: n.e.r.: $100 \%$.

2. R.: dados dos objeiós de diferente longitud, el alumno indicará bajo la consigna verbal: "pinta", "tacha", etc., "cuál es más largo" y «cuál es más corto» y reconocerá verbalmente con la consigna verbal: "¿cómo es éste?", "más corto/largo que éste».

Co.: los objetos presentados serán reales o representados en dibujos, tendrán diferente longitud y diferentes caracteristicas.

Cr.: n.e.r.: $100 \%$.

3. R.: dados tres o más objetos, el alumno indicará bajo la consigna verbal: «tacha», "subraya», etc., "el más largo de todos» y "el más corto de todos" y reconocerá verbalmente bajo la consigna verbal: "cómo es éste...» «el más largo» o «el más corto" de todos.

Co.: los objetos presentados serán reales o representados en dibujos. Tendrán diferente longitud y diferentes características.

Cr.: n.e.r.: $100 \%$.

4. R.: dados tres o más objetos y un objeto como modelo, el alumno indicará bajo la consigna verbal: "pinta», "subraya», etc., el que es el doble o la mitad de largo que el modelo, y reconocerá verbalmente bajo la consigna verbal: "¿cómo es éste?», "el doble», «la mitad de éste».

Co.: los objetos serán reales o representados en dibujos y tendrán las mismas características, aparte de la longitud, que variará según el caso.

Cr.: $80 \%$.

5. R.: dados los siguientes objetos: regla, cinta métrica y metro, cl alumno indicará bajo la consigna verbal: «señala» el objeto requerido, e indicará verbalmente el nombre y la utilidad del objeto que se le señala.

Co.: los objetos presentados serán reales o representados en dibujos.

Cr.: n.e.r.: $100 \%$.

\section{Medicionn numérica}

6. R.: dados cuatro objetos diferentes, pero de longitud correspondiente a 1 metro, el alumno reconocerá verbalmente esta longitud como de 1 metro bajo la consigna verbal: "¿qué longitud tiene esto?»

Co.: una regla de I metro, un metro articulado, una cinta métrica.

Cr.: n.e.r.: $100 \%$.

7. R.: el alumno enumera verbalmente objetos y distancias aproximadamente de I metro de largo, bajo la consigna verbal: «dime objetos que tengan 1 metro de largo».

Co.: objetos habituales del entorno.

Cr.: $80 \%$.

8. R.: dada una distancia de I centímetro, el alumno la reconocerá verbalmente bajo la consigna verbal: "¿qué longitud tiene esto?"

Co.: objetos de i centímetro, por ejemplo, regleta blanca Consinaire, uña, etc.

Cr.: n.e.r.: $100 \%$.

9. R.: el alumno enumera verbalmente objetos de aproximadamente 1 centimetro de largo, bajo la consigna verbal: "dime objetos que tengan i centímetro de largo".

Co.: objetos habituales del entorno.

Cr.: n.e.r. $80 \%$.

1o. R.: el alumno reconocerá verbalmente que I metro es más largo que I centímetro y que I centímetro es más corto que I metro, bajo las consignas verbales: "¿qué es más largo,

I metro o I centímetro?" y "¿qué es más corto, 1 metro o I centimetro?" 
Co.: el alumno no tiene a la vista los objetos que reconoce como de 1 metro y I centímetro de longitud.

11. R.: dado un conjunto de objetos, el alumno indicará bajo la consigna verbal: "señala», "rodea», etc., los que se miden en metros y los que se miden en centímetros, y enumerará verbalmente objetos que se miden en metros y objetos que se miden en centímetros bajo la consigna verbal: «dime objetos que se miden en metros/centímetros».

Co.: los objetos presentados serán reales o representados en dibujos. Los objetos para medirse en centímetros han de ser de menos de jo centímetros y los objetos para medirse en metros de más de 1 metro.

Cr.: $80 \%$.

12. R.: el alumno mide objetos familiares en metros redondeando al número de metros más cercano.

Co.: el alumno dispone de una cinta métrica. Los objetos han de tener entre 3 y 5 metros. $\mathrm{Si}$ el objeto mide, por ejemplo, 3,5 metros, el alumno puede decir 3 metros o 4 metros; si mide más de 3,5, el alumno ha de decir 4 metros; si mide menos de 3,5 metros, debe decir 3 metros.

Cr.: n.e.r. $80 \%$.

13. R.: el alumno mide objetos familiares en centimetros redondeando al número de centímetros más cercano, si es necesario.

Co.: el alumno dispone de una regla con no más de roo centímetros.

Los objetos no deben pasar de 1 metro. Los objetos serán reales.

Cr.: n.e.r. $80 \%$.

14. R.: dadas las abreviaturas $\mathrm{m}$. y $\mathrm{cm}$., el alumno las lee como metro y centímetro, respectivamente. Igualmente, dado un valor numérico, el alumno lo califica mediante m. y cm. para referirse a metros y centímetros, respectivamente.

Co.: ocasiones en las que aparezcan las abreviaturas $\mathrm{m}$. y $\mathrm{cm}$. y ocasiones en que convenga calificar un valor numérico.

Cr.: n.e.r. $100 \%$.

Is. R.: el alumno reconocerá verbalmente que hay 100 centímetros en 1 metro y que 1 metro es igual a 100 centímetros bajo la consigna verbal: «¿cuántos centímetros tiene 1 metro?», "I metro a qué es igual?"

Co.: el alumno no tiene a la vista ninguna cinta métrica o similar.

Cr.: $100 \%$.

16. R.: el alumno estima y a continuación mide, o mide objetos mayores de 1 metro en centimetros.

Co.: los objetos serán reales.

Cr.: n.e.r. $80 \%$.

17. R.: el alumno estima y a continuación mide, o mide objetos mayores de 1 metro, dando el resultado en metros y centimetros.

Co.: los objetos serán reales.

Cr.: $80 \%$.

18. R.: dada la distancia de i milímetro, el alumno la reconocerá verbalmente como tal, bajo la consigna verbal: "¿qué longitud tiene...?»

Co.: distancias en $\mathrm{I}$ milímetro y reglas milimetradas.

Cr.: $100 \%$.

19. R.: el alumno enumera verbalmente objetos de, aproximadamente, i milimetro de largo, bajo la consigna: «dime objetos que tengan 1 milímetro de largo».

Co.: objetos habituales del entorno: monedas, agujas, etc.

Cr.: $80 \%$.

20. R.: el alumno enumera verbalmente objetos que se miden en milímetros, bajo la consigna verbal: «dime objetos que se midan en milímetros». 
Co.: objetos habituales del entorno. El alumno no dispone de ningún instrumento de medida.

Cr.: $80 \%$.

21. R.: dada la abreviatura mm., el alumno la lee como milímetro. Igualmente, dado un valor numérico, el alumno lo califica mediante $\mathrm{mm}$. para referirse a milimetros.

Co.: ocasiones en las que aparezca la abreviatura mm. y ocasiones en que convenga calificar un valor numérico.

Cr.: n.e.r. $100 \%$. 
ACTIVIDADES DEL OBJETIVO NUMERO I DE LONGITUD

Situación

Profesor

Alumno

Dos objetos de la misma o diferente longitud.

¿Son iguales estos dos objetos?

Tres objetos, que aparte de la longitud, tendrán las mismas características, y dos de ellos tendrán la misma longitud.

Idem, pero con dibujos en la pizarra.

Señala los objetos que son iguales.

Une, tacha, etc..., los dos dibujos que son iguales de largo.

Idem, pero con dibujos en el Idem. papel.

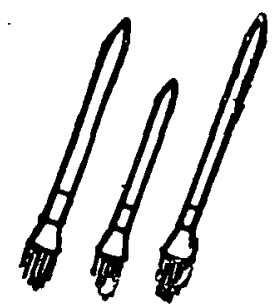

Nota: cambiar muy a menudo los dibujos y su distribución.

\section{ACTIVIDADES DEL OBJETIVO NUMERO 2 DE LONGITUD}

Situación

Profesor

Alumno

Lós objetos de diferente longitud, por ejemplo, 2 lápices.

Dibujos en el papel

a)

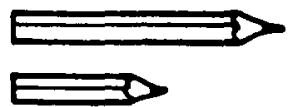

b)

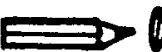

a) Señala qué lápiz es más largo.

b) Señala qué lápiz es más corto.

Señala, rodea, etc.

a) ¿Qué lápiz es más largo que el otro?

b) ¿Qué lápiz es más corto que el otro?

b)



Nota: cambiar a menudo los . dibujos de los objetos y su distribución. 
Fichas de papel con dos dibujos; al compararlos, en uno de ellos falta una parte.


Idem.

AC'TIVIDADES DEL OBJETIVO NUMERO 3 DE LONGITUD a) ¿Qué parte le falta a este dibujo?

b) Dibújala haciéndola más larga que la otra.

c) Dibújala haciéndola más corta que la otra.

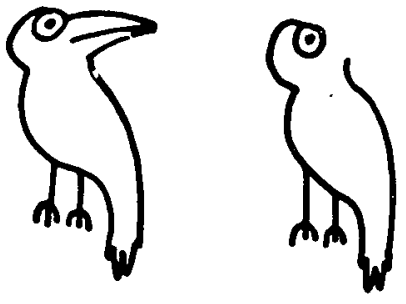 \\ $\left(\frac{1}{7 n}\right.$}

a) Responde correctamente en el ejemplo, falta el "cordel del globo".
Fichas de papel con tres dibujos, que inicialmente tendrán las mismas propiedades, excepto en la longitud, por ejemplo:

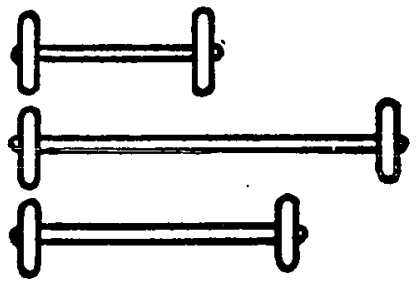

Después se pueden añadir más dibujos y de distintas propiedades entre ellos, por ejemplo:

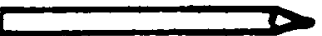

Marca con una X el más largo. Marca con una O el más corto

- Tacha el más largo de todos.

- Pinta el más corto de todos.

- Une con una línea los que son igual de largos. 
ACTIVIDADES DEL. OBJETIVO NUMERO ${ }_{4}$ DE LONGITUD

Situación

Dos trozos de cuerda, uno el doble de largo que el otro.

Dibujos en el papel, un rectángulo modelo de color rojo y tres rectángulos; la longitud de los cuales variará según cada caso, por ejemplo:
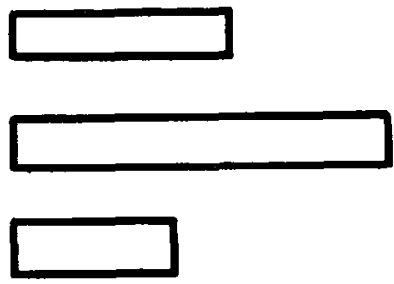

ACTIVIDADES DE LOS OBJETIVOS NUMEROS 6 y 7 DE LONGITUD
Pon una $X$ en el rectángulo

que es doble del rojo.

Idem, que es la mitad.

Idem, tan largo como.
Alumno
Ponlos juntos y compara sus longitudes; di qué trozo es el doble que el otro, y cuál es la mitad.

Los compara y responde correctamente.

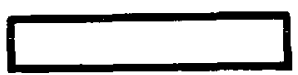

Preguntas escritas:

¿Mide más de un metro?

$$
\text { SI NO }
$$

Tu mesa

두

Un niño determinado

El educador

¿Mide menos de un metro?

Idem

Pon una cruz en el lugar correspondiente para responder SI o NO en cada pregunta.

Tu mesa

Un niño determinado

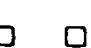

SI NO

- \

邓

(Preguntas parecidas a las anteriores).

Las mismas preguntas presentadas. oralmente por el profesor.

¿Mide más de 1 metro tu pro- Sí. fesor?
Etc. 
ACTIVIDADES DE LOS OBJETIVOS NUMEROS 6 y 7 DE LONGITUD

\begin{tabular}{|c|c|c|c|c|}
\hline Situación & Profesor & AJumno & & \\
\hline \multirow[t]{2}{*}{ Preguntas } & ¿Eres más alto que un metro? & $\begin{array}{l}\text { Debe responder } \\
\text { cada pregunta. }\end{array}$ & Si 0 & NO a \\
\hline & $\begin{array}{l}\text { ¿Eres más bajo o corto que } \\
\text { un metro? } \\
\text { ¿El metro te llega a la bar- } \\
\text { billa? } \\
\text { ¿Puedes abrir los brazos ha- } \\
\text { ciendo un metro? } \\
\text { ¿Puedes dar un paso de un } \\
\text { metro? }\end{array}$ & & & \\
\hline $\begin{array}{l}\text { Las mismas preguntas pre- } \\
\text { sentadas escritas: }\end{array}$ & $\begin{array}{l}\text { Pon la X en el lugar corres- } \\
\text { pondiente para responder SI } \\
\text { o NO a cada pregunta. }\end{array}$ & & & \\
\hline NO & & & SI & NO \\
\hline $\begin{array}{l}\text { El metro te llega } \square \\
\text { a la barbilla. }\end{array}$ & & $\begin{array}{l}\text { El metro te llega } \\
\text { a la barbilla? }\end{array}$ & $\square$ & ג \\
\hline
\end{tabular}

Etc.

\section{ACTIVIDADES DE LOS OBJETIVOS NUMEROS 6 y 7 DE LONGITUD}

Situacion

Profesor

Alumno

Material: una hoja de papel y Dibuja cosas que sean:

lápiz.
a) menos largas que $1 \mathrm{~m}$.
b) aproximadamente $1 \mathrm{~m}$.
c) más largos que $1 \mathrm{~m}$.

Dime objetos que tengan:

Ejemplos:
a) aproximadamente $1 \mathrm{~m}$.
a) el tablero de anuncios de la clase.
b) más largos que $1 \mathrm{~m}$.
b) la altura de la puerta.
c) más cortos que $1 \mathrm{~m}$.
c) el lápiz.

\section{ACTIVIDADES DE LOS OBJETIVOS NUMEROS 8 y 9 DE LONGITUD}

\begin{tabular}{ll}
\multicolumn{1}{c}{ Situación } & \multicolumn{1}{c}{ Profesor } \\
\hline & $\begin{array}{l}\text { Explica qué ancho de la uña } \\
\text { mide aproximadamente l } \\
\text { cm. }\end{array}$ \\
Material: una hoja de papel y & $\begin{array}{l}\text { Dibuja el contorno de un de- } \\
\text { do, aprovechando el dibujo }\end{array}$ \\
dépiz. & $\begin{array}{l}\text { de uña para hacer una cara. } \\
\text { Busca, aprovechando que tu } \\
\text { uña mide l cm. de ancho, } \\
\text { objetos que midan l cm. } \\
\text { aproximadamente. }\end{array}$
\end{tabular}

Alumno

$1 \mathrm{~cm}$.


Busca con la uña por la clase.

. Por ejemplo:

- El grueso de la mesa.

- Una goma de borrar, etc. 
Preguntas:

¿Cómo medirías estos obje-

tos?

Un lápiz.

Un pupitre.

Un autobús.

El libro.

Un niño.

Una casa.

También pueden presentarse estas mismas preguntas, $u$ otras similares, escritas:

Cómo medimos estos obje- Escribè cada palabra en la tos: lápiz, mesa, autobús, etc. . columna que corresponda.



\section{ACTIVIDADES DEL OBJETIVO NUMERO 2 DE LONGITUD}

Situación

Materiales: cinta métrica, metálica, metro de madera articulado.

Estimación y a continuación comprobación de ésta.

Profesor

Alumno

Mide en metros las siguientes longitudes:

- Ancho de tu mesa.

- Largo de la pizarra.

- Ancho de la pizarra.

- Ancho de la mesa del profesor.

- Ancho de la puerta.

- Altura de la puerta.

- Áncho de la ventana.

- Longitud de la habitación (aula).

- Áncho de la habitación (aula).

- Altura de la ventana.

a) ¿Cuántos metros crees que mide el ancho de la pizarra?

b) Comprueba, utilizando la cinta métrica, el ancho real de la pizarra.

(lista anterior).
Mide y da el resultado oralmente o por escrito.

a) Da el resultado oral o escrito.

b) Mide la anchura de la pizarra y da el resultado oral o escrito, comparándolo con el resultado de su estimación. 
ACTIVIDADES DEL OBJETIVO NUMERO I 3 DE LONGITUD

Sitúación

Profesor

Alumno

Matẹrial: regla de menos de

$5 n \mathrm{~cm}$ de larga

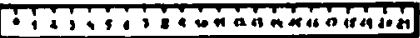

Dibujos de clempiés de longitudes exactas en $\mathrm{cm}$.


$\mathrm{cm}$.


\section{Ciming}

$\mathrm{cm}$.

Medida del cuerpo, redondeando al centímetro más cercano, si hace falta.

Material: una cinta métrica de ropa.

Lista de las partes del cuerpo a medir:

Yo tengo . . . . . cm. de alto. Mi brazo tiene ...... $\mathrm{cm}$. de largo.

Mi cuello mide . . . . . cm.

Mi cabeza mide . . . . . cm

Mi cintura mide . . . . cm

Mi muñeca mide . . . . cm.

Mi tobillo mide ...... $\mathrm{cm}$.

Material: una regla, papel y lápiz.
Mide cada ciempiés y escribe el resultado en el lugar que le corresponda.

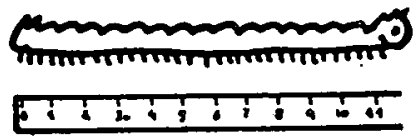

Mide tú mismo las partes de tu cuerpo, referentes a esta lista, y escribe el resuiltado en el lugar correspondiente.
Dibuja una línea de:

- $10 \mathrm{~cm}$. de largo.

- $6 \mathrm{~cm}$. de largo.

- Etc. 
ACTIVIDADES DEL OBJETIVO NUMERO I 6 DE LONGITUD

Distintos dibujos en el papel. señalando la distancia a medir con una flecha.

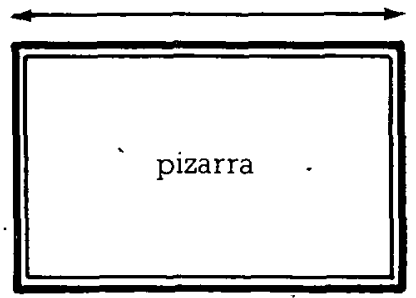

$\mathrm{m}$

cm

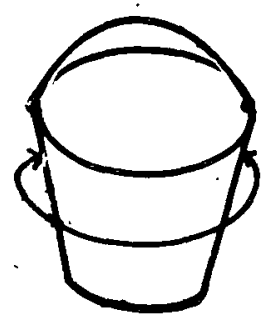

$m$.

$\mathrm{cm}$
Busca estos objetos dentro de la clase y mide cada uno de ellos, prifhero en metros y luego en centímetros.

\section{ACTIVIDADES DEL OBJETIVO NUMERO 16 DE LONGITUD}

Situación

Profesor

Alumno

Estimación y seguidamente medida en centímetros de objetos más largos de 1 metro

\section{Objeto}

La altura de la puerta.

La altura del profesor.

La longitud de la ventana.

La altura de la pizarra.

NOTA: las actividades para el objetivo $n .^{\circ} 17$ pueden ser las mismas del objetivo $n .^{\circ} 16$, pero pidiendo el resultado en $\mathrm{m}$. y en $\mathrm{cm}$ a) ¿Cuántos $\mathrm{cm}$. crees que mide la altura de la puerta?

b) Comprueba, usando la cinta métrica, la altura real. a) Dar el resultado oral o escrito.

b) Mide y da el resultado oral o bien escrito y lo compara seguidamente con el resultado de su estimación. 
ACTIVIDADES DE LOS OBJETIVOS NUMEROS 18, i9 y 20 DE LONGITUD

Situación

Material: una regla, papel y lápiz.

Preguntas.



\section{CURRICULUM ELEMENTAL DE METRICA-OBJETIVOS}

\section{Temperatura}

\section{Medición no numérica}

I. R.: Dados dos objetos que tengan igual o diferente temperatura, el alumno indicará si tienen la misma o diferente temperatura, bajo la consigna verbal: «Estos dos objetos, ¿tienen la misma temperatura?"

Co.: Los objetos serán reales o representados en dibujos.

Cr.: 100 por 100.

2. R.: Dados dos objetos que tengan diferente temperatura, el alumno indicará bajo la consigna verbal: "Señala», etc., cuál es el caliente/frío, más caliente/más frío, y reconocerá verbalmente bajo la consigna verbal: «¿Cómo está éste?», caliente/frio, más caliente/más frío. Co.: Los objetos presentados serán reales y representados en papel.

Cr.: 100 por 100. b) $2 \mathrm{~mm}$.

$15 \mathrm{~mm}$., etc.
Utilizando la regla dibuja líneas de:

- $100 \mathrm{~mm}$.

¿Cómo medirías cada cosa, en $\mathrm{m} . \mathrm{cm}$. o mm?

- Un sello.

- Una pantalla de televisión.

- El grueso de una moneda.

- El edificio de la escuela.

Hacer una relación de diferentes cosas que se puedan medir con cada una de estas

El profesor explica que escribir mm. es una forma corta para escribir milímetros.

a) Leer lo que sigue: $\begin{array}{rr}1 \mathrm{~mm} . & 5 \mathrm{~mm} . \\ 35 \mathrm{~mm} . & 50 \mathrm{~mm} .\end{array}$

b) Escribir lo que sigue de forma más corta:
ros
15 milímetros 81 milímetros.
La altura hasta la cerradu-

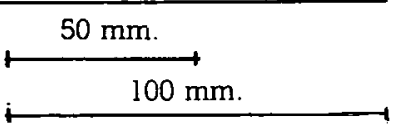


3. R.: Dados tres o más objetos teniendo diferentes temperaturas, el alumno indicará, bajo la consigna verbal: «Señala», etc., cuál es el más caliente/el más frío, y reconocerá verbalmente bajo la consigna verbal: “¿Cómo es éste?», el más caliente, el más frío.

Co.: Los objetos presentados serán reales y representados en dibujos.

Cr.: 100 por 100 .

4. R.: Dados los objetos siguientes: termómetro ordinario, termómetro para medir la temperatura del cuerpo y termómetro de máximas y mínimas, el alumno indicará, bajo la consigna verbal: "Señala» el objeto requerido e indicará verbalmente el nombre y la utilidad del objeto que se le señale.

Co.: Los termómetros presentados serán reales o representados en dibujos.

Cr.: 100 por 100 .

ร. R.: Dados dos termómetros, uno de ellos mostrando una temperatura alta y el otro una temperatura baja, el alumno indicará, bajo la consigna verbal: «Señala», «rodea con un círculo», etc., cuál representa más frío y cuál más calor, y reconocerá verbalmente que cuando la columna está más alta representa más calor y que cuando está más baja representa más frio, bajo la consigna verbal: «Cuándo está más bajo/alto, qué nos indica?» Co.: Los termómetros presentados serán reales o representados con dibujos.

Cr.: n.e.r.: 100 por 100 .

\section{Medición numérica}

6. R.: El alumno reconoce verbalmente que la unidad para medir la temperatura es el grado, bajo la consigna verbal: «¿En qué se mide la temperatura?»

Co.: - - .

Cr.: n.e.r.: ioo por ioo.

7. R.: Dado un termómetro representado en un 'dibujo, el alumno lee la temperatura que marca a intervalos de ro grados sobre o.

Co.: En el termómetro únicamente están numerados los intervalos de 10 grados.

Cr.: n.e.r.: 80 por 100.

8. R.: Dado un termómetro representado en un dibujo, el alumno lee la temperatura que marca a intervalos de 5 grados sobre 0 .

Co.: En el termómetro sólo están numerados los intervalos de, grados.

Cr.: n.e.r.: 80 por 100.

9. R.: Dado un termómetro representado en un dibujo, el alumno lee la temperatura que marca a intervalos de I grado sobre o.

Co.: En el termómetro sólo están numerados los intervalos de 5 grados.

Cr.: n.e.r.: 80 por 100.

Io. R.: El alumno enumera verbalmente temperaturas frescas y calurosas, bajo la consigna verbal: "Dime temperaturas que puedz haber cuando hace calor/frio».

Co.: - . -

Cr.: n.e.r.: 80 por 100 .

I1. R.: Ell alumno teconoce verbalmente que la temperatura de ebullición del agua es de 100 grados, bajo la consigna verbal: "¿A qué temperatura hierve el agua?»

Co.: - . -

Cr.: n.e.r.: 100 por 100 .

12. R.: El alumno reconoce verbalmente que la temperatura de congelación del agua es de - grados, bajo la consigna verbal: "¿A qué temperatura se congela el àgua?»

Co. - .

Cr.: n.e.r.: 100 por 100 .

13. R.: El alumno reconoce verbalmente que la temperatura del cuerpo es de 37 grados $(36,5)$, bajo la consigna verbal: «¿Cuál es la temperatura normal del cuerpo?»

Co.: - . -

Cr.: n.e.r.: 100 por 100. 
14. R.: Dado un objeto o un entorno, el alumno utiliza el termómetro para leer la temperatura por sobre 0 .

Co.: Un termómetro comercial normal.

Cr.: n.e.r.: 80 por 100.

Is. R.: Dado un termómetro representado en un dibujo, el alumno lee la temperatura que marca a intervalos de ro grados bajo o.

Co.: En el termómetro únicamente están numerados los intervalos de 10 grados.

Cr.: n.e.r.: 80 por 100.

16. R.: Dado un termómetro representado en un dibujo, el alumno lee la temperatura que marca a intervalos de, grados bajo o.

Co.: En el termómetro únicamente están numerados los intervalos de g grados.

Cr.: n.e.r.: 80 por 100.

17. R.: Dado un termómetro representado en un dibujo, el alumno lee la temperatura que marca a intervalos de 1 grado bajo o.

Co.: En el termómetro sólo están numerados los intervalos de grados.

Cr.: n.e.r.: 80 por 100 .

18. R.: dado un objeto o entorno, el alumno utiliza el termómetro para leer la temperatura por debajo de o.

Co.: Un termómetro comercial normal.

Cr.: n.e.r.: 80 por 100.

19. R.: El alumno reconoce la temperatura de condiciones determinadas, bajo la consigna verbal: "¿A qué temperatura estás enfermo? (40)", "¿a qué temperatura se ha de ajustar el termostato de la casa? (18/20)", etc.

Cù.: - - -

Cr.: n.e.r.: 80 por 100.

\section{ACTIVIDADES DEL OBJETIVO NUMERO 4 DE TEMPERATURA}

Situación

Preguntas y discusión con los alumnos para poder introducir el concepto y la utilidad de un termómetro.

Profesor

Alumno

La clase, ¿está fría o caliente? Fuera, en el patio, ¿hace frío o calor?

¿Cómo sabemos si hace frío o calor?

Efectivamente podemos notarlo.

¿Cómo podemos saber si fue$\mathrm{ra}$, en el patio, hace frío 0 calor sin ir al patio?

Es decir, ¿cómo podemos saber la temperatura que hace fuera, en el patio?

También podemos saber la temperatura que ha hecho, leyéndola en el peribdico o escuchándola en la T.V.

¿Como sabe el hombre del tiempo la temperatura que ha hecho?
Discusión.

Discusión.

Utilizando el termómetro.

Por qué utiliza el termómetro. 
ACTIVIDADES DEL. OBJETIVO NUMERO, DE TEMPERATURA

Material: tijeras, cartulina blanca con un termómetro dibujado, cartulina roja.
Materiales: dos botes de agua, uno de agua caliente y el otro de agua fría (cubitos de hielo).

Un termómetro.

Papel de aluminio.
Recorta el termómetro dibujado en el papel, y recorta una tira de cartulina roja, que representará la columna de mercurio del termómetro.

Cuando la tira roja está alta, ¿Cuál es la temperatura?

Y cuando está baja, ¿cuál es la temperatura?

Ahora moveré la tira roja y tú me dices si el termómetro indica una temperatura caliente o fría.

Ahora miraremos un termómetro de verdad, y me dices si ves la tira roja, azul, etc.

Tapa los botes con papel de aluminio para que el alumno no pueda ver el agua.

Pon despacio el termómetro en el agua y vigila la columna de mercurio.

¿Cuál de los dos botes tiene el agua más caliente?

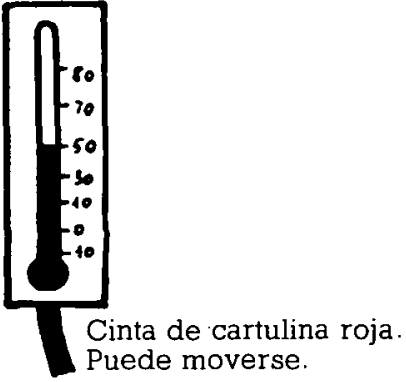

Es caliente.

Es fría

Uno a uno cogen el termómetro y lo miran, cambiándolo de posición, hasta que ven la columna de mercurio.

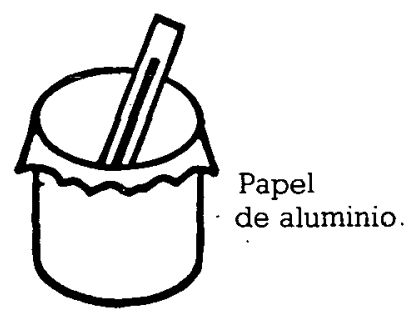

\section{ACTIVIDADES DEL OBJETIVO NUMERO 7 DE TEMPERATURA}

Situación

Termómetros representados en el papel, cada uno marca una temperatura exacta a intervalos de $10^{\circ}$ sobre 0 .


grados
Escribe la temperatura que. marca cada termómetro. 


\section{ACTIVIDADES DE LOS OBJETIVOS NUMEROS 8 y 9 DE TEMPERATURA}

Termómetros representados en el papel. Cada uno marca una temperatura exactá a intervalos de $5^{\circ}$ sobre 0 .

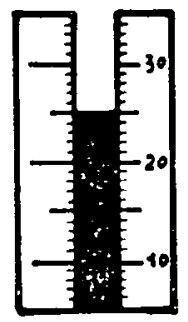

Escribe la temperatura que marca cada termómetro.

grados.

Idem, pero a intervalos de $1^{\circ}$ sobre 0
Escribe la temperatura que marca cada termómetro.

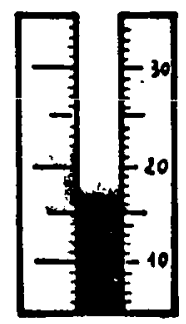

grados.

ACTIVIDADES DEL OBJETIVO NUMERO IO DE TEMPERATURA

Situación

Dibujos, fotografías de revistas, diapositivas, etc., con detalles característicos para cada una de las estaciones del año, para poder relacionar el tiempo atmosférico con la temperatura y la forma de vestir.

Idem a los materiales de antes.

Papel y lápiz.
Profesor

Alumno

A medida que las estaciones cambian, nosotros también hemos de cambiar muestra forma de vestir.

¿Qué ropa te pondrias en este tiempo (atmosférico) que indica la fotografia?

Escribe la temperatura que crees que hace en cada uno de los dibujos o fotografías.

Haz dibujos y escribe al lado la temperatura que crees corresponda a cada uno de ellos. 
ACTIVIDADES DE LOS OBJETIVOS NUMEROS I y I 2 DE TEMPERATURA

Explorar la temperatura

Materiales: un bote con cubitos de hielo. Un bote con agua hirviendo. Dos termómetros.

Dibujo en el papel:

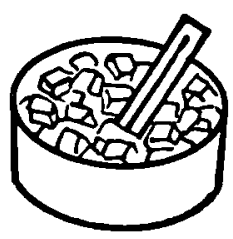

El agua se hiela a ..... grados

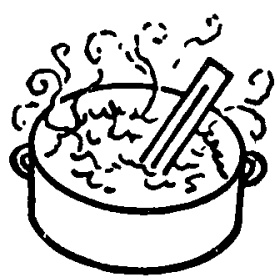

El agua hierve a .... grados.
Pon un termómetro en cada bote y dime qué temperatura alcanza cuando el agua está helada y cuando el agua está hirviendo.
El agua se hiela a $0^{\circ} \mathrm{C}$.

$\mathrm{El}$ agua hierve a $100^{\circ} \mathrm{C}$.
Escribe la temperatura que marcaria el termómetro en cada caso

\section{ACTIVIDADES DEL OBJETIVO NUMERO ${ }_{3}$ DE TEMPERATURA}

Termómetro representado en el papel.

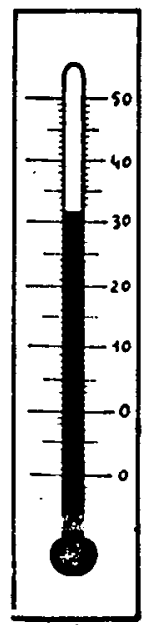

Mira el termómetro: la línea roja representa la columna de mercurio en un termómetro real, el nivel de la temperatura está entre $30^{\circ}$ y $40^{\circ}$. ¿Qué temperatura marca?

Esta es la temperatura normal de tu cuerpo. 
ACTIVIDADES DEL OBJETIVO NUMERO 14 DE TEMPERATURA

Un termómetro

CARTA DE TEMPERATURA


Un termómetro y la lista:

\begin{tabular}{l|l|l} 
Lugar & Hora & Temperatura \\
\hline & &
\end{tabular}

Se cuelga esta carta de temperatura en el tablero de anuncios de la clase.

Toma la temperatura del patio cada día de la semana, anotando la hora y los grados que marca.

Utiliza un termómetro para medir 3 temperaturas de 3 objetos o situaciones diferentes (por ejemplo, patio, aula, pasillo, etc.) y las escribes en la lista anotando también la hora de la medición.

Explica que 10 grados sobre

0 también podemos escribir-

lo de la siguiente forma: $10^{\circ}$, y que 10 grados bajo 0 podemos escribirlo asi: $-10^{\circ}$.

a) Lee lo que sigue:

$$
-20^{\circ}, 15^{\circ},-8^{\circ}, 2^{\circ}
$$

b) Escribe de una forma más simple:

15 grados bajo 0 .

12 grados sobre 0 .

6 grados bajo 0 .

b)

$$
\begin{array}{r}
15^{\circ} \\
12^{\circ} . \\
-6^{\circ} .
\end{array}
$$


ACTIVIDADES DE LOS OBJETIVOS NUMEROS Is, 16 y 17 DE TEMPERATURA

Termómetros representados en el papel. Cada uno marca una temperatura diferente sobre o bajo 0

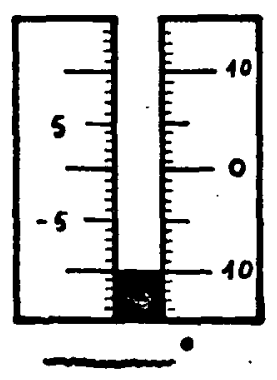

Escribe la temperatura que

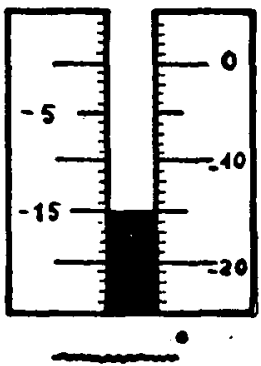

marca cada termómetro.

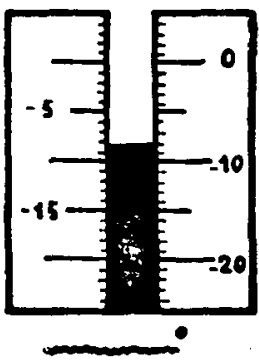


ACTIVIDADES DE LOS OBJETIVOS NUMEROS 1 , 16 y 17 DE TEMPER IIL R.I

Situación

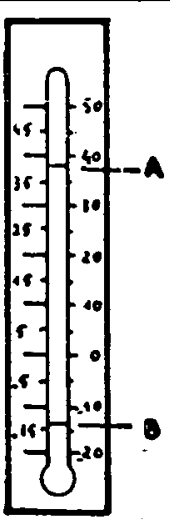

Termómetros representados en el papel

$-10^{\circ} \mathrm{C}$

$7^{\circ} \mathrm{C}$

$-18^{\circ} \mathrm{C}$, etc.
Profesor

Alumno

¿Qué temperatura marca el termómetro en el punto A y en el punto $B$ ?

Escribela en su lugar correspondiente.



A $\ldots \cdots^{\circ}$

ACTIVIDADES DE LOS OBJETIVOS NUMEROS $i 5$, I6 y 17 DE TEMPERATURA

Temperaturas escritas: $-10^{\circ} 0^{\circ}-20^{\circ} 50^{\circ}$

Temperaturas escritas: $-23^{\circ} \quad 40^{\circ}-10^{\circ} 3^{\circ}$
Rellena la línea para cada una de las temperaturas escritas, con un lápiz de color rojo.
¿Cuál de estas temperaturas es la más caliente?

Tacha o redondea en un círculo la temperatura que representa más frío. 
ACTIVIDADES DEL OBJETIVO NUMERO 9 DE TEMPERATURA

En los siguientes dibujos:


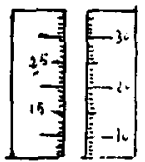

Hace sol y mucho calor y vamos en camiseta. Estamos a $30^{\circ}$.

Está nublado, llueve y hace frio. Llevamos una chaqueta. Estamos a $9^{\circ}$.

Nieva. Tenemos frío en la Idem. nariz y llevamos bufanda y

Estamos a $-7^{\circ}$

Se está bien dentro de casa. Idem. Estamos a $20^{\circ}$. gorro.

Llena la línea del termómetro hasta $30^{\circ}$.

Llena la línea del termómetro a la temperatura correspondiente.

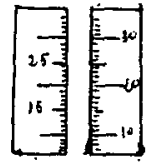

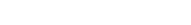




\section{CURRICULUM ELEMENTAL DE METRICA-OBJETIVOS}

\section{Capacidad}

\section{Medición no numérica}

I. R.: Presentados dos recipientes teniendo claramente la misma o distinta capacidad, el alumno indicará si tienen la misma capacidad o no, comparando directamente los volúmenes, bajo la consigna verbal: "¿En estos dos recipientes cabe igual cantidad de agua?», "¿tienen la misma capacidad o no?», etc.

Co.: Los recipientes serán de forma igual, pero de tamaño distinto.

Cr.: n.e.r.: 100 por 100.

2. R.: Presentados dos recipientes, claramente con distinta capacidad, el alumno indicará qué recipiente tiene más/menos capacidad, con la consigna verbal: “¿En cuál de los dos recipientes cabe más/menos agua?», "¿Cuál de los dos tiene más/menos capacidad?» Co.: Los recipientes serán de igual forma, pero de tamaño distinto.

Cr.: n.e.r.: 100 por 100.

3. R.: Presentados más de dos recipientes, teniendo claramente diferente capacidad, el alumno indicará cuál de ellos es el que tiene más/menos capacidad, con la consigna verbal: «¿Cuál de estos recipientes es el que tiene más/menos capacidad?"

Co.: Los recipientes serán de forma igual, pero de tamaños diferentes.

Cr.: 100 por 100.

4. R.: Presentados dos recipientes de forma igual, el alumno indicará si están igualmente llenos o no, con la consigna verbal: «Dime si estos dos recipientes están igual llenos o no».

Co.: Los recipientes tendrán la misma forma y tamaño y estarán igual llenos o no de algún líquido según convenga. También se pueden representar los recipientes y el volumen ocupádó por medio de dibujos.

Cr.: n.e.r.: 100 por 100.

5. R.: Presentados dos recipientes de forma igual, el alumno indicará cuál está más lleno/vacío, bajo la consigna verbal: «¿Cuál de estos recipientes está más lleno/vacío?», "¿cómo está este recipiente en relación con el otro?» (más lleno/más vacio).

Co.: Los recipientes tendrán la misma forma y tamaño y contendrán líquido a distintos niveles.

6. R.: Presentados más de dos recipientes de forma igual, el alumno indicará qué recipiente está más lleno/vacío, bajo la consigna verbal: «¿Cuál de estos recipientes es el que está más lleno/vacío?"

Co.: Los recipientes deberán tener la misma forma y tamaño y contendrán líquido a distintos niveles.

Cr.: n.e.r.: 100 por 100.

7. R.: Presentados más de dos recipientes de capacidades claramente diferentes, el alumno los ordenará de mayor a menor capacidad o viceversa, bajo la consigna verbal: “Ordena estos recipientes de mayor a menor capacidad."

Co.: Los recipientes serán de forma igual, pero de tamaños diferentes.

Cr.: n.e.r.: Ioo por 100.

8. R.: Presentados más de dos recipientes de forma igual, el alumno los ordenará de más lleno a menos o viceversa, bajo la consigna verbal: «Ordena estos recipientes del que está más lleno al más vacío.»

Co.: Los recipientes serán de la misma forma y tamaño y estarán llenos de líquido a distintos niveles.

Cr.: n.e.r.: 100 por 100.

\section{Medición numérica}

9. R.: Presentado un recipiente de 1 litro de capacidad, el alumno reconocerá verbalmente 
esta capacidad como unidad estándar para medir líquidos y volúmenes, bajo la consigna verbal: «¿Qué capacidad tiene este recipiente?»

Co. - .

Cr.: n.e.r.: 100 por 100.

Io. R.: El alumno identifica recipientes familiares, la capacidad de los cuales se aproxima a I litro, bajo la consigna verbal: "Dime cosas que tengan I litro de capacidad" o "Dime cosas en las cuales quepa i litron.

Co.: Recipientes fáciles de encontrar y conocidos de capacidad aproximada a 1 litro. Cr.: n.e.r.: 80 por 100 .

11. R.: Presentados varios recipientes de formas diferentes y un recipiente unidad de i litro de capacidad, el alumno mide las capacidades de todos tomando el número de unidadeslitro correspondiente al volumen total de cada recipiente, bajo la consigna verbal: «Mide la capacidad de este recipiente» o «¿cuántos litros caben. en este recipiente?» Co.: - . -

Cr.: n.e.r.: 8o por 100 .

12. R.: Presentado un recipiente cualquiera, el alumno estimará su capacidad en términos de unidades-litro e inmediatamente verificará su estimación. mediante el procedimiento descrito en el objetivo anterior.

Co.: - -

Cr.: n.e.r.: 80 por ıoo.

13. R.: Presentados dos recipientes de capacidad distinta, el alumno mide sus capacidades respectivas, nombrándolas en términos unidades-litro, y compara los resultados.

Co.: Los recipientes tendrán formàs y volúmenes diferentes ò de formas diferentes y volúmenes iguales.

Cr.: n.e.r.: 80 por 100 .

14. R.: Dada la abreviatura 1., el alumno la lee como litro. Igualmente, dado un valor numérico, el alumno lo califica mediante l. para referirse a litros.

Co.: Ocasiones en que aparezca la abreviatura 1 . y ocasiones en que convenga calificar un valor numérico.

Cr.: n.e.r.: 100 por 100

\section{CURRICULUM ELEMENTAL DE METRICA-OBJETIVOS}

\section{Peso}

\section{Medición no numérica}

1. R.: Presentados dos objetos de peso claramente distinto o igual, el alumno indicará si son iguales o diferentes comparando sus pesos directamente, bajo la consigna verbal: " $i$ Tienen el mismo peso?", "ipesan igual?»

Co.: Objetos reales de pesos claramente iguales o diferentes.

Cr.: n.e.r.: 100 por 100.

2. R.: Presentados dos objetos de pesos claramente diferentes, el alumno indicará qué objeto es más pesado/ligero, bajo la consigna verbal: "¿Cuál de estos dos objetos es más pesado/ligero?" y reconocerá verbalmente con la pregunta verbal: “¿Cómo es este objeto, más pesado/ligero?»"

Co.: Objetos reales de pesos claramente diferentes.

Cr.: n.e.r.: ioo por 100 .

3. R.: Presentados más de dos objetos de pesos diferentes, el alumno indicará qué objeto es el más pesado/ligero, bajo la consigna verbal: "¿Cuál de estos objetos es el más pesado/ligero?" y reconocerá verbalmente, bajo la consigna verbal: "¿Cómo es este objeto, el más pesado/ligero?».

Co.: Objetos reales de pesos distintos.

Cr.: n.e.r.: 100 por 100 . 
4. R.: Presentados más de dos objetos de pesos diferentes, el alumno los ordenará del más pesado a menos o viceversa, bajo la consigna verbal: «Ordena estos objetos de más pesados a menos" o viceversa.

Co.: Objetos reales de pesos distintos.

Cr.: n.e.r.: 100 por 100.

\section{Medición numérica}

5. R.: Presentado un objeto de peso igual a i kilo, el alumno reconocerá verbalmente este peso como la unidad estándar para medir el peso, bajo li consigna verbal: «¿Qué peso tiene este objeto?»"

Co.: El objeto puede ser cualquiera o también una pesa de I kilo.

Cr.: n.e.r.: 100 por 100 .

6. R.: El alumno identifica objetos familiares, el peso de los cuales se aproxima a a kilo, bajo la consigna verbal: "Dime cosas que pesen más o menos I kilo».

Co.: Objetos fáciles de encontrar y. conocidos de peso aproximado de 1 kilo. Cr.: n.e.r.: 80 por 100 .

7. R.: Presentados distintos objetos de pesos diferentes, el alumno mide su peso en kilos, redondeando al kilo más próximo.

Co.: El alumno dispone de una báscula.

Cr.: n.e.r.: 80 por 100.

8. R.: Presentado un objeto cualquiera, el alumno estimará su peso en términos de unidades-kilo e inmediatamente verificará su estimación mediante el procedimiento descrito en el objetivo anterior.

Co.: -. -

Cr.: n.e.r.: 80 por 100 .

9. R.: Presentados dos objetos de pesos difereńtes, el alumno medirá su peso respectivo, expresándolo en términos de unidades-kilo, y comparará los resultados.

Co.: El alumno dispone de una báscula para efectuar las medidas.

Cr.: n.e.r.: 80 por 100.

10. R.: Presentado un objeto de peso igual a un gramo, el alumno lo reconocerá verbalmente, bajo la consigna verbal: «¿Qué peso tiene este objeto?»

Co.: Objetos familiares y conocidos de peso aproximado a I gramo.

Cr.: n.e.r.: 100 por 100.

I I. R.: El alumno enumera verbalmente objetos de peso aproximado a I gramo, bajo la consigna verbal: «Dime cosas que pesen aproximadamente I gramo".

Co.: Objetos familiares y conocidos de peso aproximado a i gramo.

Cr.: n.e.r.: 80 por 100.

12. R.: El alumno, dados una serie de objetos de menos de I kilo, los medirá en gramos redondeando al gramo más cercano, bajo la consigna verbal: «Dime el peso de este objeto». Co.: El alumno dispone de una báscula.

Cr.: n.e.r.: 80 por 100 .

13. R.: Dado un objeto cualquiera de menos de I kilo, el alumno estimará su peso en gramos e inmediatamente verificará su estimación mediante el procedimiento descrito en el objetivo anterior.

Co.: - -

Cr.: n.e.r.: 80 por 100.

14. R.: El alumno reconocerá verbalmente que 1 kilo es más pesado que 1 gramo y que 1 gramo es menos pesado (o más ligero) que i kilo, bajo las consignas verbales: «¿Qué pesa más, I kilo o I gramo?" y "¿qué pesa menos, I kilo o I gramo?"

Co.: El alumno no tiene a la vista los objetos que reconoce como i kilo y i gramo. Cr.: n.e.r.: 100 por 100. 
1 s. R.: Dado un conjunto de objetos, el alumno indicará, bajo la consigna verbal: «Rodea con un circulo", "tacha", etc., los que se miden en kilos y los que se miden en gramos, y enumerará verbalmente objetos que se midan en kilos y objetos que se midan en gramos, bajo la consigna verbal: «Dime objetos que se midan en kilos/gramos».

Co.: Los objetos presentados serán reales o representados en dibujos. Los objetos para medir en gramos han de ser de menos de 1 kilo y los objetos para medir en kilos han de ser de peso superior a i kilo.

Cr.: n.e.r.: 80 por 100.

16. R.: Dadas las abreviaturas $\mathrm{Kg}$. y g., el alumno las lee como kilo y gramo, respectivamente. Igualmente, dado un valor numérico, el alumno los califica mediante $\mathrm{Kg}$. y g. para - referirse a kilos y gramos, respectivamente.

Co.: Ocasiones en las que aparezcan las abreviaturas $\mathrm{Kg}$. y g. y ocasiones en que convenga calificar un valor numérico.

Cr.: n.e.r.: 100 por 100 .

17. R.: El alumno reconocerá verbalmente que hay $1.000 \mathrm{~g}$. en $1 \mathrm{Kg}$. y que $1 \mathrm{Kg}$. es igual a

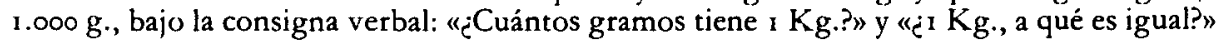
Co.: - -

Cr.: n.e.r.: 1.00 por 100 .

\section{Referencias}

Borelli, M. L. Morelli, S. Z.: Teaching Measurement to Children: Grades k-6. Revised Edition. ED $171547,1979$.

Elementay Metric Curriculum-Project T.I.M.E. (Timely Implementation of Metric Education) Part I. ED $171556,1979$.

Elementary Metric Curriculum-Project T.I.M.E. (Timely Implementation of Metric Education) Part II. ED 171 1 $57,1979$.

Metrics: The Future Is Now. ED 171 555, 1979. 